\title{
Identification of a mutL-homolog 1 mutation via whole-exome sequencing in a Chinese family with Gardner syndrome
}

\author{
ZILAN LV $^{1 *}$, CHUAN WANG $^{2 *}$, LIXIANG WU $^{1}$, BIANQIN GUO $^{1}$, DARONG ZHANG $^{1}$, \\ YANG ZHANG $^{1}$, SHENGXING HUANG ${ }^{3}$ and MINGLIN OU ${ }^{4,5}$ \\ ${ }^{1}$ Department of Laboratory Medicine, Chongqing University Cancer Hospital and Chongqing Cancer Institute \\ and Chongqing Cancer Hospital, Chongqing 400030; ${ }^{2}$ Department of Gastroenterology, \\ Chongqing General Hospital, Chongqing 400014; ${ }^{3}$ Medical Center of Stomatology; ${ }^{4}$ Clinical Medical Research Center, \\ The Second Clinical Medical College, Jinan University, Shenzhen People's Hospital, Shenzhen, Guangdong 518020; \\ ${ }^{5}$ Guangxi Key Laboratory of Metabolic Diseases Research, Central Laboratory of \\ Guilin No. 181 Hospital, Guilin, Guangxi 541002, P.R. China
}

Received September 9, 2017; Accepted February 12, 2018

DOI: $10.3892 / \mathrm{mmr} .2018 .9063$

\begin{abstract}
Gardner syndrome (GS), a variant of familial adenomatous polyposis, is a rare genetic disorder with autosomal dominant inheritance, characterized by the presence of multiple intestinal polyps, multiple osteomas, dental abnormalities and soft tissue tumors. To date, only a few gene mutations have been demonstrated to be responsible for GS. To explore potential unknown mutations responsible for GS, the present study used whole-exome sequencing of two affected individuals from a family with GS to identify a candidate mutation in mutL-homolog (MLH)1. The two patients with GS were diagnosed based on a combination of clinical features, family history, physical examinations and cone-beam computed tomographic imaging. Through whole-genome sequencing, the present study subsequently identified a missense mutation in MLH1 (NM_000249.3:p.Tyr379Ser/c.1136A>C), which was further confirmed by Sanger sequencing. Furthermore, the amino acid residue p.Tyr379 was identified to be highly conserved among different species through sequence
\end{abstract}

Correspondence to: Dr Minglin Ou, Clinical Medical Research Center, The Second Clinical Medical College, Jinan University, Shenzhen People's Hospital, 1017 Dongmen North Road, Shenzhen, Guangdong 518020, P.R. China

E-mail: minglinou@163.com

Professor Shengxing Huang, Medical Center of Stomatology, The Second Clinical Medical College, Jinan University, Shenzhen People's Hospital, 1017 Dongmen North Road, Shenzhen, Guangdong 518020, P.R. China

E-mail: shxhuang@sina.com

${ }^{*}$ Contributed equally

Key words: gardner syndrome, dental abnormalities, whole-exome sequencing, mutation, mutL-homolog 1 alignment with ClustalW2. In conclusion, the results identified for the first time a MLH1 missense mutation (NM_000249.3: p.Tyr379Ser/c.1136A>C) in a Chinese family with GS, thus broadening the range of mutated genes associated with GS. This highlights the value of whole-exome sequencing in identifying disease mutations in a family.

\section{Introduction}

Gardner's syndrome (GS) is considered to be a variant form of familial adenomatous polyposis. In the early 1950s, GS was described by Gardner as a rare genetic disorder with autosomal dominant inheritance (1). GS affects approximately $10 \%$ of individuals with familial adenomatous polyposis (2). The primary clinical features of the syndrome are diffuse adenomatous polyposis, multiple osteomas, dental abnormalities and soft-tissue tumors (3). The intestinal polyps typically develop at $\sim 20$ years of age and have up to a $100 \%$ potential for malignant transformation following 10-20 years, whereas dental anomalies precede the intestinal polyps and are present in $30-75 \%$ of patients with GS $(1,4)$. The dental anomalies may include impacted or un-erupted teeth, congenitally missing teeth, hypercementosis, supernumerary teeth, dentigerous cysts, long and tapered molar roots, fused molar roots, hypodontia or compound odontomas $(3,5)$. Osteomas are present in $68-82 \%$ of GS patients and are generally located in the mandible and paranasal sinuses (6).

A series of mutations in certain genes, including adenomatous polyposis coli (APC), mutY DNA glycosylase, mutL homolog (MLH)1, mutS homolog (MSH)2, MSH6, PMS1 homolog (PMS)2, birt-hogg-dube homolog, serine/threonine kinase 11, bone morphogenetic protein receptor type 1A, mothers against decapentaplegic homolog 4 and phosphatase and tensin homolog, have been reported to be associated with hereditary gastrointestinal polyposis syndromes (7). At present, only a few gene mutations associated with GS have been reported, probably due to fewer patients suffering from this disease. Namely, mutations in MYH and APC, including 
various different mutations in APC, have been associated with GS (8-10).

With the development of next-generation sequencing technology, whole-exome sequencing is now used to detect exome variant profiles, and is considered a powerful and cost-effective tool due to its identification of extensive disease-associated variations in novel genes $(11,12)$. Therefore, the present study assessed two members of a Chinese family with GS using exome sequencing to screen for the disease-causing gene mutation. From analysis of the exome sequencing data, the present study first identified an MLH1 missense mutation (NM_000249.3:p.Tyr379Ser/c.1136A>C) in the family members with GS, which was subsequently confirmed by Sanger sequencing. The reported mutation may be valuable for the prenatal and genetic diagnosis of GS.

\section{Materials and methods}

Subjects. The present study recruited a Chinese Han family in which two family members (the father and the son) presented with GS in August, 2016. The father was 52-years-old, and the son were 26-years-old. The diagnosis of GS was based on clinical features, family history, cone-beam computed tomography (CBCT), colonoscopy and pathological examinations. Genomic DNA was extracted from peripheral venous blood from the two affected individuals using a QIAamp DNA Blood Mini kit (Qiagen GmbH, Hilden, Germany) following the manufacturer's protocol. Informed consent was obtained from each patient involved in the study, and the study protocol was approved by the Ethics Committee of Guangxi Key Laboratory of Metabolic Diseases Research (Guilin, China).

Whole-exome sequencing. Genomic DNA was isolated from the peripheral venous blood of the patients using the QIAamp DNA Blood Mini kit (Qiagen $\mathrm{GmbH}$ ) and then used for exome capture using a NimbleGen SeqCap EZ Human Exome Library v2.0 kit (Roche NimbleGen Inc., Wisconsin, USA) following the manufacturer's protocol. Random DNA fragmentation was performed with a Covaris Ultrasonicator system (Covaris Inc., Woburn, MA, USA), after which the sizes of the library fragments were mainly distributed between 150 and 250 bp. An 'A' base was added at the 3'-end of each strand, then ligated to sequencing adapters, which was followed by ligation-mediated polymerase chain reaction (PCR) with probe hybridization, amplification and purification to enrich for targets to sequence. Primers used were included in the NimbleGen SeqCap EZ Human Exome Library v2.0 kit. Each resulting qualified captured library then was sequenced on a BGISEQ-500 sequencing platform (Beijing Genomics Institute, Guangdong, China) and the desired average sequencing coverage for each sample was obtained. Raw image files were processed to produce pair-end reads for each individual using default parameters of base calling software developed for BGISEQ-500.

Read mapping and variant analysis. Clean data was obtained by raw data filtering, and the clean data of each sample was mapped to the human reference genome (GRCh37/HG19) using a Burrows-Wheeler Aligner (BWA V0.7.15) $(13,14)$. All genomic variations, including single-nucleotide polymorphisms (SNPs) and insertions/deletions (InDels) were identified using
HaplotypeCaller of GATK (v3.3.0) (Broad Institute, Cambridge, MA, USA) with the proper filtering parameters $(15,16)$. Subsequently, the SnpEff tool (http://snpeff.sourceforge .net/SnpEff $\backslash$ _manual.html) was used to perform a series of annotations for variants. Potential disease-causing mutations were predicted using the sorting intolerant from tolerant (SIFT) algorithm (17). If the SIFT score was $\leq 0.05$, the present study predicted this variant to bea deleterious variant. Datawere filtered with several variant databases, including dbSNP (https://www .ncbi.nlm.nih.gov/projects/SNP/), the 1000 Genomes Project (ftp://ftp-trace.ncbi.nih.gov/1000genomes/ftp/release), and the NHLBI-ESP6500 database (http://evs.gs.washington .edu/EVS/). Candidate mutations were expected to be absent from these databases. The conservation analysis of amino acid sequences were aligned using ClustalW2 (http://www.ebi.ac.uk/Tools/msa/clustalw2/).

Sanger sequencing. Sanger sequencing was used to confirm candidate mutations in the MLH1 gene identified by exome sequencing. The PCR primers used were as follows: Forward, 5'-CTTAGTACTGCTCCATTTGGGGA-3' and reverse, 5'-TTGTTGTATCCCCCTCCAAGC-3'. PCR amplification was performed using a HEMA 9600 PCR thermo cycler (Technological Innovation Beach, Zhu Hai, Guangdong, China) with 35 cycles of denaturation at $98^{\circ} \mathrm{C}$ for $10 \mathrm{sec}$, annealing at $55^{\circ} \mathrm{C}$ for $30 \mathrm{sec}$ and extension at $72^{\circ} \mathrm{C}$ for $30 \mathrm{sec}$. The PCR reagents, including Taq polymerase, Taq polymerase buffer, $\mathrm{MgCl}_{2}$ and $\mathrm{dNTP}$ mixture were purchased from Takara Biotechnology, Co., Ltd. (Dalian, China). The PCR products were sequenced using an ABI Prism 3730 DNA analyzer (Applied Biosystems; Thermo Fisher Scientific, Inc., Waltham, MA, USA). Each read was compared with the genomic DNA sequence of MLH1, and nucleotide alterations were numbered according to their position in MLH1.

\section{Results}

Clinical data. Clinical data was collected for the two patients through patient interviews, medical record extraction, physical examinations and CBCT imaging.

The proband (II:1; Fig. 1) of the GS family, a 52-year-old male patient, presented at the hospital with swelling and pain in the mandibular region, and was diagnosed with GS. From his medical history, it was discovered that the patient had already undergone intestinal polypectomy 3 years ago with recurrence. He had presented with swelling and pain in the mandibular region 2 years previously, and had undergone surgical debridement following anti-inflammatory treatment. A pathological diagnosis of 'ossified fibromatosis with infection' was made. The patient continued to present with swelling and discomfort repeatedly, and underwent curative jaw osteomyelitis surgery in the other hospital a year prior to the most recent presentation. Family history revealed that the patient's father had died from gastrointestinal cancer. At the clinical examination, the patient demonstrated no abnormal skin pigmentation, skin mass or swelling in the liver and spleen. On intraoral examination, it was observed that the patient's lower teeth on the right side were missing since surgery, a swelling was present in the right mandibular buccal side, a swelling with pus was present in the mucosal layer, the deciduous teeth 54, 55, 65 and 
I

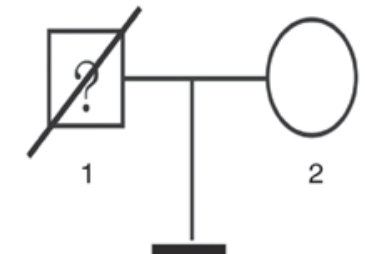

II

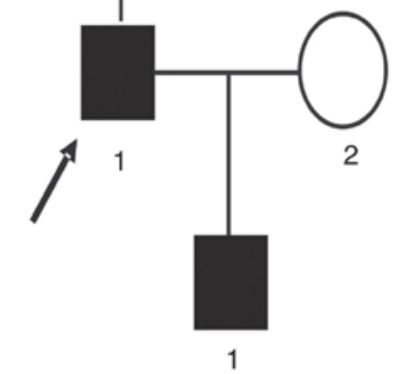

Figure 1. Pedigree of the family with Gardner syndrome (the arrow indicates the proband).
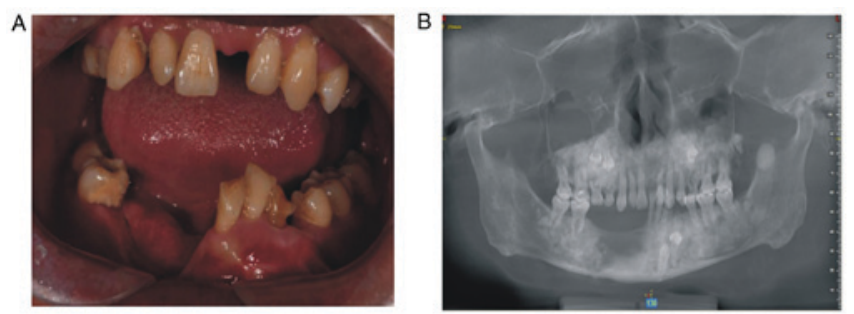

C

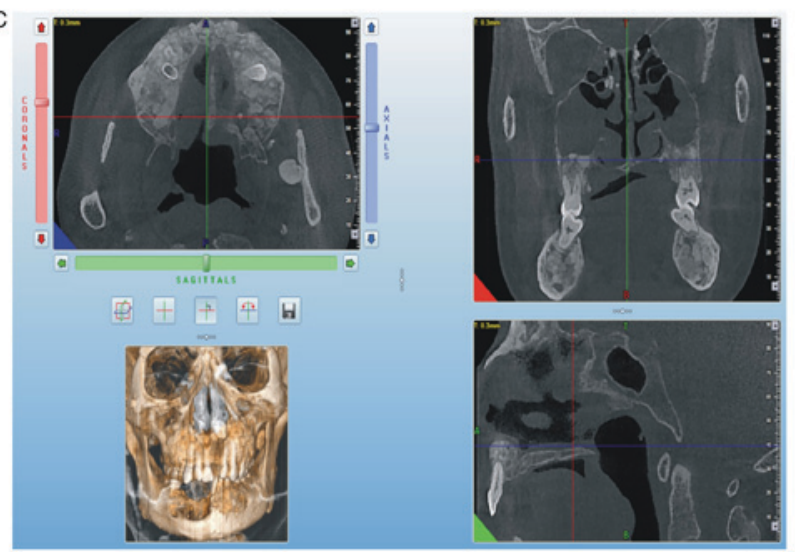

Figure 2. Oral examination of the proband (II:1). (A) Intraoral photograph. (B) Panoramic image. (C) Cone-beam computed tomographic images.

73 were retained and tooth 34 was unerupted (Fig. 2A). The panoramic radiograph (Fig. 2B) and CBCT revealed that there was an increase in irregular density in the upper and lower jaw regions, and the retained deciduous teeth corresponding to permanent teeth $14,15,25,33$ and 34 were affected, and that multiple convex hyperplastic bone lesions were present in the right upper and lower jaw and ethmoidal sinus (Fig. 2C).

The son of the proband (III:1; Fig. 1), a 26-year-old male, requested a clinical assessment due to his father being diagnosed with GS. Medical history revealed that the son had repeatedly abdominal fibrous tumors lasting for 10 years and intestinal polyps lasting for 3 years. At clinical examination, the patient exhibited a semicircular elevated region due to a tough, fixed mass with clear boundaries on the right abdominal skin, $\sim 15 \mathrm{~cm}$ in diameter (Fig. 3A). On intraoral examination,
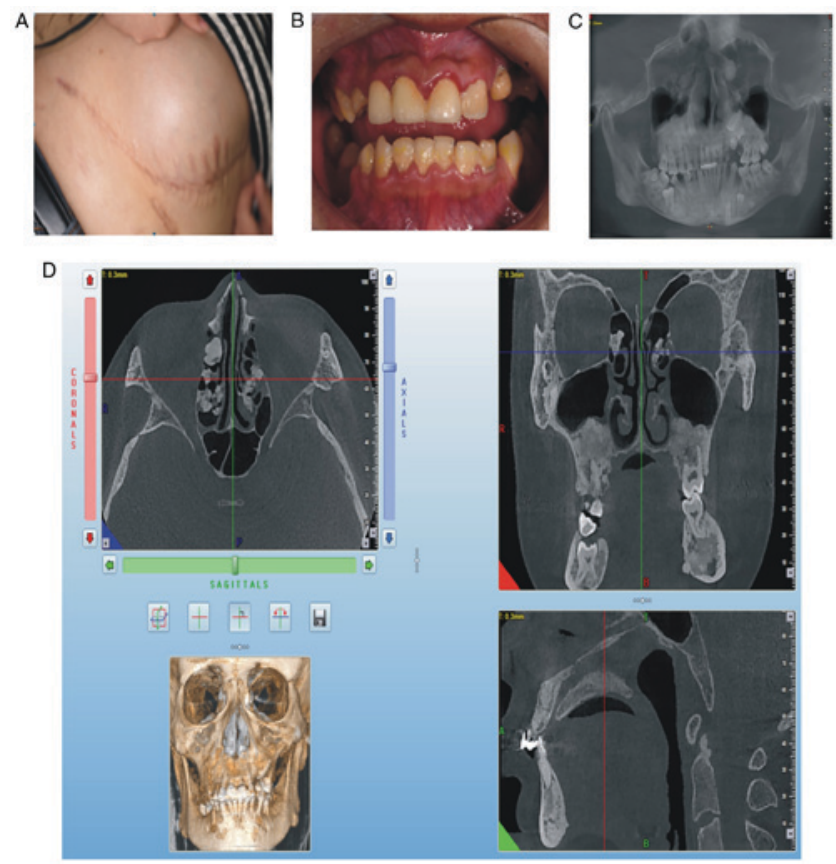

Figure 3. Oral examination of the affected member (III:1). (A) A photograph of the patient's abdomen. (B) Intraoral photograph. (C) Panoramic image. (D) Cone-beam computed tomographic images.

it was revealed that deciduous teeth 53, 63, 73 and 83 were retained, whereas teeth 25,35 and 46 were erupted (Fig. 3B). The panoramic radiograph (Fig. 3C) and CBCT indicated that permanent teeth $13,23,25,33,35$ and 46 were impacted, that supernumerary teeth were not present, that there was an increase in irregular density in the upper and lower jaw regions, and that multiple convex hyperplastic bone lesions were present in the ethmoid sinus (Fig. 3D).

Whole-exome sequencing. The present study performed whole-exome sequencing on both affected individuals (II:1 and III:1) in the GS Chinese family, for which an average of 31,766.99 Mb raw bases were screened. Following removal of low-quality reads, an average of 31,753.08 Mb clean reads were obtained. The average GC content was $54.26 \%$. Total clean reads per sample were aligned to the human reference genome (GRCh37/HG19) using BWA. On average, 99.05\% mapped successfully. The duplicate reads were removed, resulting in an average of 20,694.12 Mb effective reads. The mean sequencing depth of target regions was 232.41-fold. On average, per individual sequenced, $99.86 \%$ of targeted bases were covered by at least 1x coverage while $95.09 \%$ of the targeted bases had at least 10x coverage (Table I). Overall, 112,819 single nucleotide polymorphisms (SNPs) across both individuals were identified. Of these variants, $96.79 \%$ were represented in dbSNP and $94.31 \%$ were annotated in the 1000 Genomes Project database. The number of novel SNPs was 2,967. The ratio of transition to transversion was 2.33. Of the total SNPs, 12,736 were synonymous, 11,716 were missense, 37 were stop-loss, 96 were stop-gain, 24 were start-loss and 105 were splice site mutations. In total, 16,829 InDels were detected across all samples. Of these variants, $76.89 \%$ were represented in dbSNP and $57.26 \%$ were annotated in the 1000 Genomes Project database. The number of novel InDels was 3,422. Of the overall InDels, 
Table I. Summary of whole exome sequencing data.

\begin{tabular}{lccr}
\hline & \multicolumn{2}{c}{ Pedigree ID } & Average \\
\cline { 2 - 3 } Parameter & \multicolumn{1}{c}{ II:1 } & III:1 & $635,339,711$ \\
\hline Raw reads & $613,323,244$ & $657,356,178$ & 31766.99 \\
Raw bases (Mb) & 30666.16 & 32867.81 & 31753.08 \\
Clean bases (Mb) & 30652.62 & 32853.54 & 54.26 \\
GC rate (\%) & 53.17 & 55.34 & $20,694.12$ \\
Total effective reads (Mb) & $19,086.35$ & $22,301.89$ & 99.05 \\
Mapping rate on genome (\%) & 98.97 & 99.13 & 232.41 \\
Average sequencing depth on target & 199.95 & 264.86 & 99.86 \\
Fraction of target covered>=1x (\%) & 99.98 & 99.73 & 95.09 \\
Fraction of target covered>=10x (\%) & 99.47 & 90.71 & \\
\hline
\end{tabular}

Table II. Summary statistics for identified SNPs and InDels.

\begin{tabular}{lccc}
\hline & \multicolumn{2}{c}{ Pedigree ID } & Overall \\
\cline { 2 - 4 } Parameter & II: 1 & III:1 & 112,819 \\
\hline Total number of SNPs & 99,562 & 91,132 & 96.79 \\
Fraction of SNPs in dbSNP (\%) & 96.18 & 96.40 & 94.31 \\
Fraction of SNPs in 1,000 genomes (\%) & 92.45 & 92.56 & 2,967 \\
Novel & 3,258 & 2,764 & 2.33 \\
Ti/Tv & 2.31 & 2.33 & 12,736 \\
Synonymous & 11,149 & 10,999 & 11,716 \\
Missense & 10,424 & 10,179 & 105 \\
Splicing & 101 & 90 & 16,829 \\
Total number of Indels & 13,968 & 11,050 & 344 \\
Frameshift & 274 & 291 & 169 \\
Non-frameshift Insertion & 138 & 147 & 192 \\
Non-frameshift Deletion & 149 & 141 & 58 \\
Splicing & 53 & 54 & \\
\hline
\end{tabular}

SNP, single nucleotide polymorphism; InDels, insertions/deletions.

344 were frameshift, 4 were stop-loss, 3 were start-loss and 58 were splice site mutations (Table II).

Through read mapping and variant analysis, the presence of previously known mutations in APC and MYH in the two patients was ruled out. It was observed that a novel missense mutation in the MLH1 gene (NM_000249.3:p. Tyr379Ser/c.1136A $>$ C) was present in both affected individuals, and absent in the dbSNP, 1000 Genomes Project, and NHLBI-ESP6500 databases.

Sanger sequencing. To verify the mutation in the MLH1 gene that was identified using whole-exome sequencing, Sanger sequencing was used to examine the MLH1 gene of the two patients (II:1 and III:1). Using method, it was revealed that a missense mutation in the MLH1 gene (NM_000249.3: p.Tyr379Ser/c.1136A $>$ C) was present in both affected individuals (Fig. 4). Therefore, combining the clinical

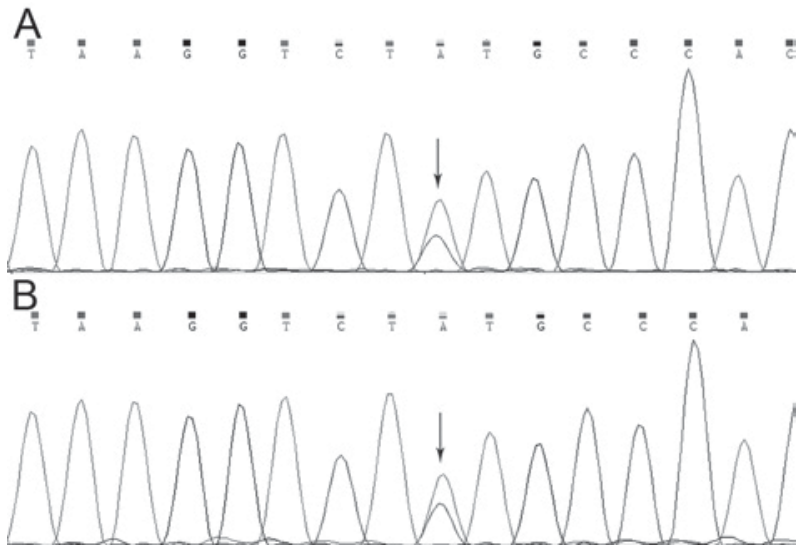

Figure 4. Sequencing analysis of the MLH1-1136 (arrows indicate the mutations). (A) Missense mutation of the proband (II:1) in MLH1 (NM_000249.3: p.Tyr379Ser/c.1136A>C). (B) Missense mutation of the son (III:1) in MLH1 (NM_000249.3:p.Tyr379Ser/c.1136A>C). 
CLUSTAL 0 (1.2.4) multiple sequence aligment

Human

Pan troglodytes

Pongo abelii

Mus musculus

Danio rerio

Solanum lycopersicum

Saccharomyces cerevisiae
EDK---TLAFKMNGYISNANYSVKKCI-FLLFINHRLVESTSLRKAIETVYAAYLPKNTH MNGYISNANYSVKKCI-FLLFINHRLVESTSLRKAIETVYAAYLPKNTH EDK---TLAFKMNGYISNANYSVKKCI-FLLFINHRLVESTSLRKAIETVYAAYLPKNTH EDK---TLAFKMNGYISNANYSVKKCI-FLLFINHRLVESAALRKAIETVYAAYLPKNTH EDQ----KFAFKVKGYISNANYSVKKCI-LILFINHRLVESSALKKAIETVYTAYLPKNTH SDTGPLISVFKMDGFISNSNYIAKKTT-MVLFINDRLIDCGALKRAIEIVYTATLPKASK SKVEDLN-LERVDGKVCNLNFISKKSISPIFFINNRLVTCDPLRRALNSVYSNYLPKGNR

Figure 5. Conservation analysis of the MLH1 p.Tyr379 amino acid residue.

data, whole-exome sequencing data, and the literature on GS, it was suggested that the MLH1 gene mutation may be responsible for GS.

Conservation analysis of MLH1 p.Tyr379. MLH1 amino acid sequences from the species Homo sapiens, Pan troglodytes, Pongo abelii, Mus musculus, Danio rerio, Solanum lycopersicum and Saccharomyces cerevisiae were aligned, and found that p.Tyr379 was located within a highly conserved region of MLH1, and was revealed to be highly conserved among the different species, which indicated the structural and functional importance (Fig. 5).

\section{Discussion}

GS, an autosomal dominant disease, has high penetrance and variable expression that may present as intestinal polyposis or extracolonic manifestations, including osteomas, skin and soft tissue tumors, dental anomalies and congenital hypertrophy of the retinal pigment epithelium. The incidence rate of GS ranges from 1 in 12,000 to 1 in 4,000, depending on the region worldwide (18).

Intestinal polyps in GS may affect the entire gastrointestinal tract. They typically start to develop during puberty and fully emerge between the ages of 20 and 40 years. The lesions have up to a $100 \%$ potential for malignant transformation generally in the 40-50-year age group (19). Due to the high potential for transformation to adenocarcinoma, the polyps must be completely removed in order to effectively prevent colon cancer. Additionally, desmoid tumors are a common manifestation of GS and are usually locally aggressive, non-malignant and non-encapsulated (20). These lesions appear in 3.5-5.7\% of GS cases and are three times more common in women (21). Desmoid tumors frequently occur in the first 3 years following colon surgery in the abdominal wall and/or intra-abdominal cavity, as observed in the second patient in the present study, who exhibited desmoid tumors in the intra-abdominal cavity. GS diagnosis is based on the presence of osteomas, and three or more lesions are typically present in $26-46 \%$ of patients (22).

In the present study, panoramic radiograph and CBCT revealed that the two patients had several dental abnormalities including multiple impacted teeth in the mandible, maxilla and ethmoidal sinus; however, surgery was performed only in the first patient (II:1), who had ossifying fibrosis with infection, resulting in swelling and pain in the mandibular region. The two patients also had a family history of GS. Therefore, the present study performed whole-exome sequencing and a large number of variants in each individual were initially identified.
Several mutations in GS family members were identified by bioinformatic filtering and segregation analysis of the data. It was concluded that the same variations in both affected individuals were likely the cause of GS.

MLH1 is a primary component of DNA mismatch repair (MMR) protein complexes, and heterodimerizes with PMS2 to form MutL $\alpha$. In this way, MMR serves an important role in genome stability $(23,24)$. MLH1 is located on 3p21.3-23, and is commonly dysregulated in colon cancer (25). It has been previously reported that the MLH1 mutation may be responsible for $50 \%$ of cases of Lynch syndrome (also known as hereditary non-polyposis colon cancer, HNPCC) (26). Acquired defects in MLH1 have been observed in 13-15\% of sporadic colorectal cancers (CRCs) (27), and the lifetime risk of contracting CRC is as high as $68 \%$ in MLH1 mutation carriers (28). It has been documented that there are numerous mutation sites in the MLH1 gene responsible for HNPCC (28), however whether the mutation site identified in the present study (NM_000249.3:p.Tyr379Ser/c.1136A>C) results in HNPCC has not been reported. Due to this, the present study focused on GS, and whether the mutation site results in HNPCC will be investigated in future work. Depending on the feature of the disease, the present study firstly sequenced MLH1 in peripheral blood from these patients, and in the future, may sequence MLH1 in tissue samples. To verify that this MLH1 mutation results in GS syndrome, the author's will perform further functional analyses in the future.

In conclusion, the present study screened for mutations in the MLH1 gene in a Chinese family with GS using whole-exome sequencing, and the findings were confirmed by Sanger sequencing. The two affected individuals in the family harbored a missense mutation in the MLH1 gene (NM_000249.3:p.Tyr379Ser/c.1136A>C) and shared a number of symptoms, including osteomas, skin and soft tissue tumors and dental anomalies. The data also demonstrated that the amino acid residue of p.Tyr379 was highly conserved among different species. Therefore, it was predicted that the p.Tyr379 mutation may impact on the proper function of MLH1 and thus may be associated with the development of GS in this family. Additionally, the present study demonstrated that whole-exome sequencing is a time- and cost-efficient method of screening and identifying gene mutations in GS. To the best of the author's knowledge, the present results may be the first to identify the MLH1 missense mutation (NM_000249.3: p.Tyr379Ser/c.1136A>C) in a Chinese family with GS, which may aid in determining genetic diagnosis and subsequent therapeutic regimens for this family. 


\section{Acknowledgements}

Not applicable.

\section{Funding}

The present study was supported by Guangxi Key Laboratory of Metabolic Diseases Research (grant no. 2016-181h-03), and by Guangxi Natural Science Foundation (grant no. 2015GXNSFBA139176).

\section{Availability of data and materials}

The datasets used and/or analyzed during the current study are available from the corresponding author on reasonable request.

\section{Authors' contributions}

MO made substantial contributions to the design and data analysis of the present study, ZL participated in experiment of the present study and data analysis, and drafted the manuscript. SW made substantial contributions to sample collection and clinical data analysis. CW participated in DNA sequencing and mutation analysis, LW, BG, DZ and YZ participated in whole-exome sequencing data analysis. All authors read and approved the final manuscript.

\section{Ethics approval and consent to participate}

The present study was approved by the Ethics Committee of Guangxi Key Laboratory of Metabolic Diseases Research. Written informed consent was obtained from all patients.

\section{Consent for publication}

Written informed consent for the publication of any associated data and accompanying images was obtained from each participant.

\section{Competing interests}

The authors declare that they have no competing interests.

\section{References}

1. Cankaya AB, Erdem MA, Isler SC, Cifter M, Olgac V, Kasapoglu C and Oral CK: Oral and maxillofacial considerations in Gardner's Syndrome. Int J Med Sci 9: 137-141, 2012.

2. Ramaglia L, Morgese F, Filippella M and Colao A: Oral and maxillofacial manifestations of Gardner's syndrome associated with growth hormone deficiency: Case report and literature review. Oral Surg Oral Med Oral Pathol Oral Radiol Endod 103: e30-e34, 2007

3. Ponti G, Tomasi A, Manfredini M and Pellacani G: Oral mucosal stigmata in hereditary-cancer syndromes: From germline mutations to distinctive clinical phenotypes and tailored therapies. Gene 582: 23-32, 2016.

4. Klein OD, Oberoi S, Huysseune A, Hovorakova M, Peterka M and Peterkova R: Developmental disorders of the dentition: An update. Am J Med Genet C Semin Med Genet 163C: 318-332, 2013.

5. Singh K, Singh A, Kumar P and Gupta N: Prosthodontic management of a patient with Gardner's syndrome: A clinical case report Dent Res J (Isfahan) 11: 276-280, 2014

6. Madani M and Madani F: Gardner's syndrome presenting with dental complaints. Arch Iran Med 10: 535-539, 2007.
7. Aretz S: The differential diagnosis and surveillance of hereditary gastrointestinal polyposis syndromes. Dtsch Arztebl Int 107: 163-173, 2010.

8. Davies DR, Armstrong JG, Thakker N, Horner K, Guy SP, Clancy T, Sloan P, Blair V, Dodd C, Warnes TW, et al: Severe Gardner syndrome in families with mutations restricted to a specific region of the APC gene. Am J Hum Genet 57: 1151-1158, 1995.

9. Juhn E and Khachemoune A: Gardner syndrome: Skin manifestations, differential diagnosis and management. Am J Clin Dermatol 11: 117-122, 2010.

10. Gu GL, Wang SL, Wei XM and Bai L: Diagnosis and treatment of Gardner syndrome with gastric polyposis: A case report and review of the literature. World J Gastroenterol 14: 2121-2123, 2008.

11. Yang Y, Muzny DM, Reid JG, Bainbridge MN, Willis A, Ward PA, Braxton A, Beuten J, Xia F, Niu Z, et al: Clinical whole-exome sequencing for the diagnosis of mendelian disorders. N Engl J Med 369: 1502-1511, 2013.

12. Bamshad MJ, Ng SB, Bigham AW, Tabor HK, Emond MJ, Nickerson DA and Shendure J: Exome sequencing as a tool for Mendelian disease gene discovery. Nat Rev Genet 12: 745-755, 2011.

13. Li H and Durbin R: Fast and accurate long-read alignment with Burrows-Wheeler transform. Bioinformatics 26: 589-595, 2010.

14. Li H and Durbin R: Fast and accurate short read alignment with Burrows-Wheeler transform. Bioinformatics 25: 1754-1760, 2010.

15. DePristo MA, Banks E, Poplin R, Garimella KV, Maguire JR, Hartl C, Philippakis AA, del Angel G, Rivas MA, Hanna M, et al: A framework for variation discovery and genotyping using next-generation DNA sequencing data. Nat Genet 43: 491-498, 2011.

16. Mckenna A, Hanna M, Banks E, Sivachenko A, Cibulskis K, Kernytsky A, Garimella K, Altshuler D, Gabriel S, Daly M and DePristo MA: The Genome Analysis Toolkit: a MapReduce framework for analyzing next-generation DNA sequencing data. Genome Res 20: 1297-1303, 2010.

17. Ng PC and Henikoff S: SIFT: Predicting amino acid changes that affect protein function. Nucleic Acids Res 31: 3812-3814, 2003.

18. Basaran $\mathrm{G}$ and Erkan M: One of the rarest syndromes in dentistry: Gardner syndrome. Eur J Dent 2: 208-212, 2008.

19. Jaiswal AS, Balusu R and Narayan S: Involvement of adenomatous polyposis coli in colorectal tumorigenesis. Front Biosci 10: 1118-1134, 2005.

20. Chung J, Namkoong S, Jung KE, Park JW, Park BC, Cinn YW and Kim MH: A case of gardner's syndrome associated with desmoid tumor. Ann Dermatol 22: 418-421, 2010.

21. Fotiadis C, Tsekouras DK, Antonak is P, Sfiniadakis J, Genetzakis M and Zografos GC: Gardner's syndrome: A case report and review of the literature. World J Gastroenterol 11: 5408-5411, 2005.

22. Herford AS, Stoffella E and Tandon R: Osteomas involving the facial skeleton: A report of 2 cases and review of the literature. Oral Surg Oral Med Oral Pathol Oral Radiol 115: e1-e6, 2013.

23. Lu Y, Wajapeyee N, Turker MS and Glazer PM: Silencing of the DNA mismatch repair gene MLH1 induced by hypoxic stress in a pathway dependent on the histone demethylase LSD1. Cell Rep 8: 501-513, 2014.

24. Kadyrova LY and Kadyrov FA: Endonuclease activities of MutL $\alpha$ and its homologs in DNA mismatch repair. DNA Repair (Amst) 38: 42-49, 2016.

25. Bronner CE, Baker SM, Morrison PT, Warren G, Smith LG, Lescoe MK, Kane M, Earabino C, Lipford J and Lindblom A: Mutation in the DNA mismatch repair gene homologue hMLH1 is associated with hereditary non-polyposis colon cancer. Nature 368: 258-261, 1994.

26. Ryan E, Sheahan K, Creavin B, Mohan HM and Winter DC: The current value of determining the mismatch repair status of colorectal cancer: A rationale for routine testing. Crit Rev Oncol Hematol 116: 38-57, 2017.

27. Hinrichsen I, Ernst BP, Nuber F, Passmann S, Schäfer D, Steinke V, Friedrichs N, Plotz G, Zeuzem S and Brieger A: Reduced migration of MLH1 deficient colon cancer cells depends on SPTAN1. Mol Cancer 13: 11, 2014.

28. Cruz-Correa M, Pérez-Mayoral J, Dutil J, Echenique M, Mosquera R, Rivera-Román K, Umpierre $\mathrm{S}$, Rodriguez-Quilichini S, Gonzalez-Pons M, Olivera MI, et al: Hereditary cancer syndromes in Latino populations: Genetic characterization and surveillance guidelines. Hered Cancer Clin Pract 15: 3, 2017. 